ACCEPTED FOR PUblication in ApJ

Preprint typeset using LATEX style emulateapj v. 04/20/08

\title{
EMBEDDED YOUNG STELLAR OBJECT CANDIDATES IN THE ACTIVE STAR FORMING COMPLEX W51: MASS FUNCTION AND SPATIAL DISTRIBUTION
}

\author{
Miju Kang ${ }^{1,2,3}$, John H. Bieging ${ }^{3}$, Matthew S. Povich ${ }^{4,5}$, And Youngung LeE ${ }^{1}$ \\ Accepted for publication in ApJ
}

\begin{abstract}
We present 737 candidate Young Stellar Objects (YSOs) near the W51 Giant Molecular Cloud (GMC) over an area of $1.25^{\circ} \times 1.00^{\circ}$ selected from Spitzer Space Telescope data. We use spectral energy distribution (SED) fits to identify YSOs and distinguish them from main-sequence or red giant stars, asymptotic giant branch stars, and background galaxies. Based on extinction of each YSO, we separate a total of 437 YSOs associated with the W51 region from the possible foreground sources. We identify 69 highly embedded Stage 0/I candidate YSOs in our field with masses $>5 M_{\odot}$ (corresponding to mid- to early-B main-sequence spectral types) 46 of which are located in the central active star forming regions of W51A and W51B. From the YSOs associated with W51, we find evidence for mass segregation showing that the most massive YSOs are concentrated on the W51 H II region complex. We find a variation in the spatial distribution of the mass function (MF) of YSOs in the mass range between $5 M_{\odot}$ and $18 M_{\odot}$. The derived slopes of the MF are -1.26 and -2.36 in the active starforming region and the outer region, respectively. The variation of the MF for YSOs embedded in the molecular cloud implies that the distribution of stellar masses in clusters depends on the local conditions in the parent molecular cloud.
\end{abstract}

Subject headings: H II regions — stars: formation — ISM: individual (W51) — infrared: ISM

\section{INTRODUCTION}

Stars form in various environments. High-mass stars are especially important because they affect their environment through such phenomena as outflows, stellar winds, and supernovae. To understand the interaction of stars and the interstellar medium (ISM) it is necessary to identify embedded young stellar objects (YSOs) in the parent molecular cloud. Therefore taking a complete census of YSOs is an important step toward understanding YSOs and their environment on a large scale.

W51 is an active star-forming region located in the Sagittarius spiral arm. The distance is uncertain, with published values including $5.1_{-1.4}^{+2.9} \mathrm{kpc}$ ( $\mathrm{Xu}$ et al. 2009 ), $6.1 \pm 1.3 \mathrm{kpc}$ (Imai et al. 2002), or $8.3 \pm 2.5$ $\mathrm{kpc}$ (Schneps et al. 1981). The radio continuum sources comprising W51 are within a massive giant molecular cloud (GMC) (Mufson \& Liszt 1979). Observations showing the star forming activity have been carried out in various wavelengths of radio continuum, HI line, molecular lines, water masers, near-IR, X-ray (Bieging 1975; Carpenter \& Sanders 1998; Imai et al. 2002; Koo 1997; Koo \& Moon 1997; Koo 1999; Mehringer 1994; Okumura et al. 2000). W51 is a region of ongoing massive star formation (Lacy et al. 2007; Zapata et al. 2008) and therefore a good place to study the feedback between

\footnotetext{
${ }^{1}$ International Center for Astrophysics, Korea Astronomy and Space Science Institute, Hwaam 61-1, Yuseong, Daejeon 305-348, South Korea; mjkang@kasi.re.kr

2 Department of Astronomy and Space Science, Chungnam National University, Daejeon 305-764, South Korea

${ }^{3}$ Steward Observatory, University of Arizona, 933 North Cherry Avenue, Tucson, AZ 85721

${ }^{4}$ Department of Astronomy, University of Wisconsin at Madison, 475 N. Charter Street, Madison, WI 53706

${ }^{5}$ NSF Astronomy and Astrophysics Postdoctoral Fellow, Department of Astronomy and Astrophysics, Pennsylvania State University, 525 Davey Lab, University Park, PA 16802
}

stars and the ISM.

To understand the interaction of molecular clouds and YSOs in the W51 $\mathrm{H}$ II region complex we mapped a $1.25^{\circ}$ $\times 1.0^{\circ}$ region in the $J=2-1$ transitions of ${ }^{12} \mathrm{CO}$ and ${ }^{13} \mathrm{CO}$ with the $10 \mathrm{~m}$ Heinrich Hertz Telescope (HHT) on Mount Graham, Arizona. The molecular line maps will be presented in a separate paper (Bieging et al., in preparation). In the present paper, we use Spitzer data for identifying the YSO candidates associated with the molecular clouds. Spitzer surveys in the mid-IR allow sensitive studies of deeply embedded star formation. Although extinction is significantly reduced in the mid-IR, it is still important for active star forming regions in giant molecular clouds. In particular, the W51 region shows no detectable increase in star counts because of severe extinction (Benjamin et al. 2005). Therefore we have to consider extinction effects carefully in identifying and characterizing candidate YSOs. Combining the YSOs selected from the Spitzer photometry and molecular clouds with kinematic information, we can examine the feedback process between star(s) and ISM. The detailed analysis for the interaction of YSOs and associated molecular clouds will be presented in other publications (Kang et al. (2009); Kang et al., in preparation). In this paper, we tabulate all of the YSO candidates and discuss their spatial distribution and mass function. In Section2, we introduce the data sets used and describe our methods for selecting and classifying candidate YSOs. We discuss some of the implications of our results in Section 3 and summarize the results in Section 4

\section{YSO SELECTION AND CLASSIFICATION}

\subsection{Data}

The Galactic Legacy Infrared Mid-Plane Survey Extraordinaire (GLIMPSE I; Benjamin et al. 2003) survey covered the Galactic plane $\left(10^{\circ}<|l|<65^{\circ},|b|<1^{\circ}\right)$ 


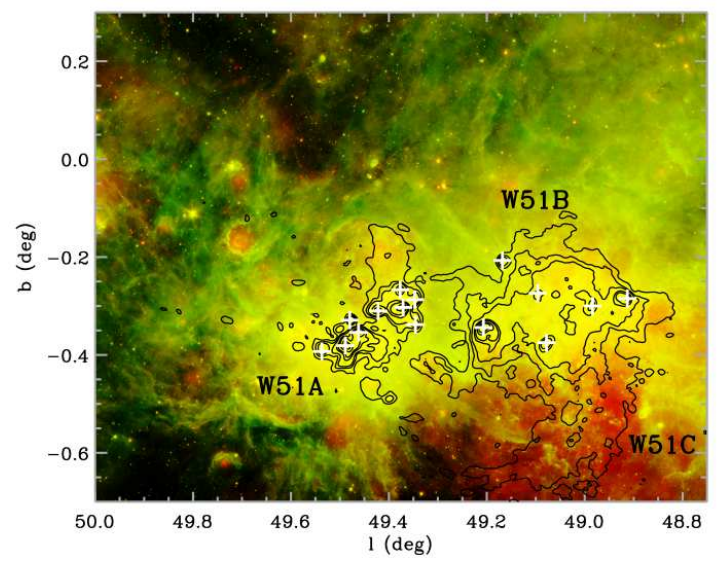

FIG. 1. - A color image of the W51 complex composed of MIPS $24 \mu \mathrm{m}$ (red) and IRAC $8.0 \mu \mathrm{m}$ (green). Hot dust grains and PAH dominated emission appear in red and green, respectively. The contours show $21 \mathrm{~cm}$ radio continuum emission made by combining VLA data and Effelsberg 100-m telescope data (Koo \& Moon 1997). The contour levels are $-0.015,0.015,0.05,0.10,0.3,0.5$, $1.0,1.5$, and $2.4 \mathrm{Jy}$ beam $^{-1}$. Cross symbols mark the radio continuum sources listed by Koo \& Moon (1997).

with the four mid-IR bands $(3.6,4.5,5.8$, and $8.0 \mu \mathrm{m})$ of the Infrared Array Camera (IRAC; Fazio et al. 2004) on the Spitzer Space Telescope. Each IRAC band contains different spectral features : Band 1 [3.6] shows mainly continuum emission from stars, Band $2[4.5]$ traces $\mathrm{H}_{2}$ rotational transitions arising in the shocked gas associated with outflows, and emission in Bands 3 [5.8] and $4[8.0]$ is dominated by polycyclic aromatic hydrocarbon (PAH) features. For this study, we have retrieved images of the $1.25^{\circ} \times 1.0^{\circ}$ region of W51 centered on $(l, b)=$ $\left(49.375^{\circ},-0.2^{\circ}\right)$ (note that this is the region we mapped in $\mathrm{CO}$ ) by combining two mosaic images which have a high resolution $\left(1^{\prime \prime} .2\right.$ pixel $\left.^{-1}\right)$ in all four IRAC bands (Figure 11). For this same region, we used the GLIMPSE I Catalog including the sources detected at least twice in one band with a $\mathrm{S} / \mathrm{N} \geq 5$ and at least once with a $\mathrm{S} / \mathrm{N}$ $>3$ in an adjacent band. Generally, the flux density limits insure that the detections are $>5 \sigma$. The $5 \sigma$ pointsource detection limits of the GLIMPSE are nominally $0.2,0.2,0.4$, and $0.4 \mathrm{mJy}$ for the IRAC 3.6, 4.5, 5.8, and $8.0 \mu \mathrm{m}$ bands, respectively. These limits are significantly higher in regions of bright diffuse emission. The GLIMPSE I Catalog also tabulates $J H K_{s}$ flux densities from the 2MASS point source catalog (Skrutskie et al. 2006) for all GLIMPSE sources with 2MASS identifications.

MIPSGAL (Carev et al. 2005) is a legacy program covering the inner Galactic plane, $10^{\circ}<|l|<65^{\circ}$ for $|b|<$ $1^{\circ}$, at 24 and $70 \mu \mathrm{m}$ with the Multiband Imaging Photometer for Spitzer Space Telescope (MIPS; Rieke et al. 2004). The resolution of the $24 \mu \mathrm{m}$ mosaics from the MIPSGAL survey is 2 .' 4 pixel $^{-1}$. We extracted $24 \mu \mathrm{m}$ point-sources with $F / \delta F>7$, then bandmerged the 24 $\mu \mathrm{m}$ sources with the GLIMPSE Catalog sources using a $2^{\prime \prime} 0$ correlation radius. The final source list consists of all 8 bands combined: 2MASS, GLIMPSE, and MIPSGAL $24 \mu \mathrm{m}$. A total of 104,582 sources within the $1.25^{\circ} \times 1.0^{\circ}$ region of W51 centered on $(l, b)=\left(49.375^{\circ},-0.2^{\circ}\right)$ were selected from the GLIMPSE I Catalog.
TABLE 1

Source Counts in the W51 Region ${ }^{\mathrm{a}}$

\begin{tabular}{lr}
\hline \hline Sources & Number \\
\hline In GLIMPSE Catalog & 104,582 \\
Fit with SED models $\left(N_{\text {data }} \geq 4\right)$ & 70,767 \\
Well-fit by stellar photosphere SEDs $\left(\chi^{2} / N_{\text {data }} \leq 4\right)$ & 69,316 \\
Poorly-fit by stellar photosphere SEDs $\left(\chi^{2} / N_{\text {data }}>4\right)$ & 1,451 \\
Well-fit by YSO SEDs $\left(\chi^{2} / N_{\text {data }} \leq 4\right)$ & 1,327 \\
In final sample of YSO candidates & 737 \\
\hline a The $1.25^{\circ} \times 1.0^{\circ}$ region centered on $(1, \mathrm{~b})=\left(49.375^{\circ},-0.2^{\circ}\right)$
\end{tabular}

\subsection{Color Selection}

The colors of most main sequence (MS) stars are near zero for any combination of the IRAC and MIPS bands, while YSOs have red colors due to the emission from surrounding warm dust. As an initial approach, we applied a well defined color selection criteria of Simon et al. (2007) to our source list for identifying YSO candidates associated with the molecular cloud around W51. After selecting candidate YSOs from the region in color-color space defined by Simon et al. (2007), we classified them with the spectral index $\alpha$ in the IRAC bands. The spectral index is

$$
\alpha=\frac{d \log [\lambda F(\lambda)]}{d \log \lambda}
$$

where $\lambda$ is the wavelength and $F(\lambda)$ is the flux at that wavelength. We obtain $\alpha$ from a linear fit to the logarithm of the fluxes for all 4 IRAC bands and then classify YSOs as Class I sources with $0.3 \leq \alpha_{I R A C}$, " Flat " sources with $-0.3 \leq \alpha_{I R A C}<0.3$, Class II sources with $-1.6 \leq \alpha_{I R A C}<-0.3$, and Class III sources with $\alpha_{I R A C}<-1.6$ (Lada 1987; Greene et al. 1994).

Figure 2( $a$ ) shows the distribution of YSOs within the color selection criteria of Simon et al. (2007) represented by the solid line on the IRAC [3.6] - [4.5] vs. [4.5] - [8.0] color-color diagram. In Figure $2(a)$, a large number of YSO candidates with red colors were not selected because the Simon et al. (2007) color criteria were designed to identify a relatively clean sample of YSOs. Many reddened main sequence stars in our W51 field were identified as YSOs, however, because the Simon et al. (2007) color criteria were derived from the Small Magellanic Cloud which is not affected by the large interstellar extinction of the Galactic plane. The W51 region is several kpc from the Sun in the Galactic plane so interstellar extinction is significant. Figure $2(b)$ shows the IRAC [3.6] - [4.5] vs. [5.8] - [8.0] color-color diagram by Simon et al. (2007) with YSO categories based on $\alpha$ for comparing with the distribution of YSOs found by other selection criteria in the same color-color space.

Figure 2( $(c)$ shows the distribution of YSOs identified with the IRAC color criteria of Gutermuth et al. (2008). They classified the YSO candidates into two groups after removing sources dominated by PAH emission, shock emission and broad-line AGNs. One group is Class I protostars and the other is the more evolved Class II sources. Applying color classification of Gutermuth et al. (2008), we found that the YSOs candidates in the W51 region separate into 366 Class I sources and 1457 Class II sources. Although these color criteria selected the interesting YSO candidates having very red colors, the sample also contained a large number of reddened stellar photo- 

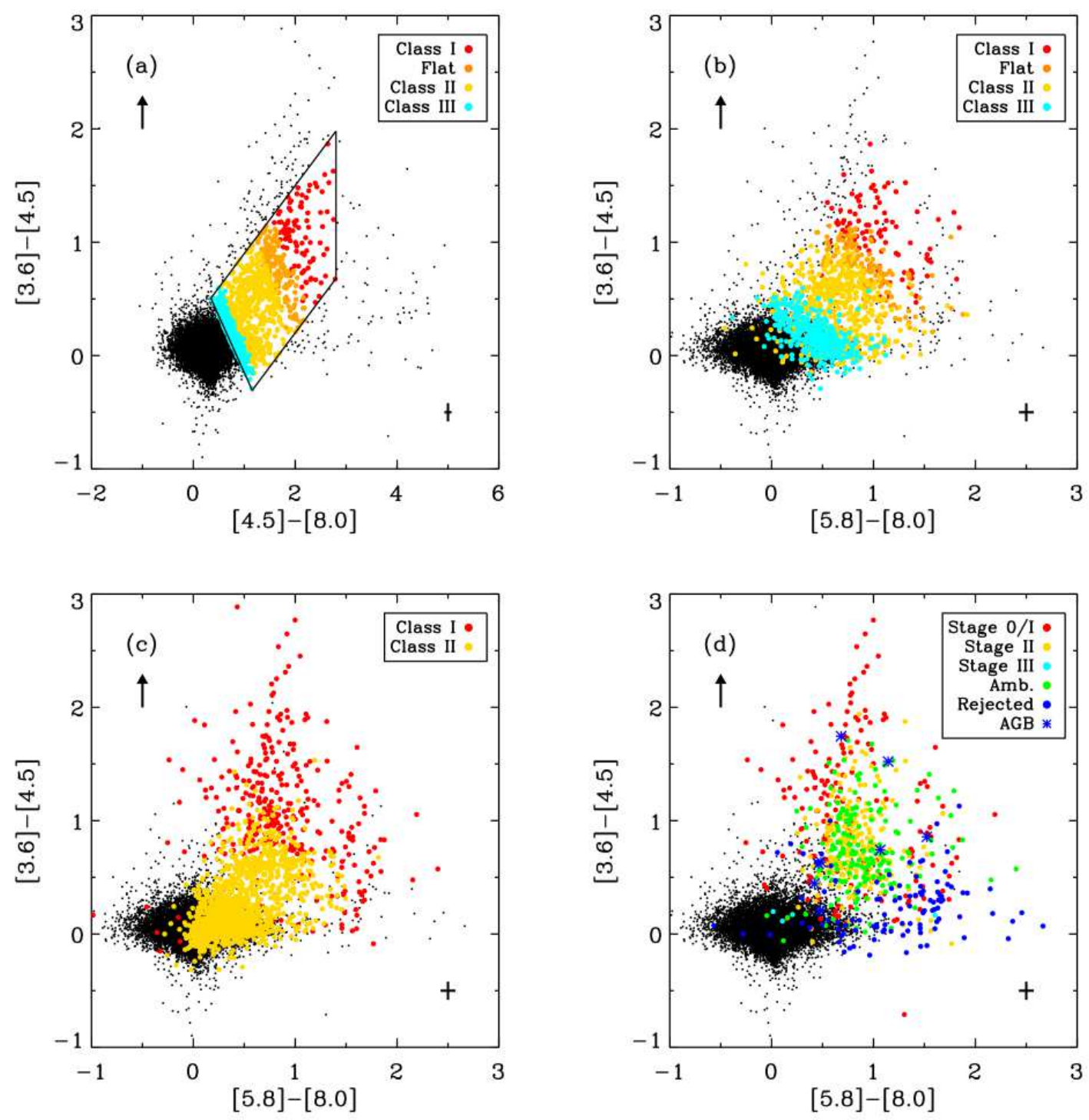

FIG. 2.- (a) IRAC [3.6] - [4.5] vs. [4.5] - [8.0] color-color diagram by the color selection criteria of Simon et al. (2007). All sources were detected in all 4 IRAC bands in the GLIMPSE Catalog. YSO candidates classified by the spectral index $\alpha$ are marked in red for Class I objects, orange for the flat-spectrum sources, yellow for the Class II sources, and cyan for the Class III sources. The solid line represents the color selection criteria of Simon et al. (2007). (b) IRAC [3.6] - [4.5] vs. [5.8] - [8.0] color-color diagram by the color selection criteria of Simon et al. (2007). (c) IRAC [3.6] - [4.5] vs. [5.8] - [8.0] color-color diagram by the color selection criteria of Gutermuth et al. (2008). Class I objects are marked in red and Class II objects are marked in yellow. (d) IRAC [3.6] - [4.5] vs. [5.8] - [8.0] color-color diagram with YSO candidates identified using the SED fitting tool. Small black dots are sources well-fit by stellar photosphere SEDs. YSO candidates are marked in red for Stage 0/I, yellow for Stage II, cyan for Stage III, and green for ambiguous sources. AGB candidates are marked by blue asterisk symbols. Blue dots are well-fit YSO candidates that were discarded from the final sample. Reddening vector for $\mathrm{A}_{V}=20$ mag based on the extinction laws of Indebetouw et al. (2005) is shown as a filled arrow. Black crosses show typical photometric errors.

spheres because the color criteria were derived from the study of the nearby star forming region, NGC 1333.

Class I, Flat, and Class II sources in Figure[2(b) belong to Class I and relatively red Class II samples in Figure 2(c). Color selection criteria may be suitable as a method to select the objects in a relatively young stage. However, more evolved sources, which include the Class III objects in Figure $2(b)$ and those Class II objects around zero color in Figure 2(c), may be mis-identified because of the large interstellar extinction toward the W51 region.

\subsection{SED fitting}

We used the spectral energy distribution (SED) fitting tools of Robitaille et al. (2007) for identifying and classifying YSOs in the W51 giant molecular cloud complex. The SED fitting tool works as a regression method to find the SEDs within a specified $\chi^{2}$ from a large grid of models after fitting the input data points. The grid of models contains stellar atmospheres, a limited set of spectra of galaxies, AGB stars, and YSO models. The grid of YSO models was computed by Robitaille et al. (2006) using the 20,000 2-D radiation transfer models from Whitney et al. (2003a, b, 2004). Each YSO model has SEDs for 10 viewing angles (inclinations), so the total YSO grid consists of 200,000 SEDs. The SED 
TABLE 2

Magnitudes OF YSO CANDidates

\begin{tabular}{|c|c|c|c|c|c|c|c|c|c|c|}
\hline \multirow[t]{2}{*}{ No. } & \multirow[t]{2}{*}{ IRAC designation } & \multicolumn{8}{|c|}{ Magnitudes } & \multirow[t]{2}{*}{ Assoc. $^{\mathrm{a}}$} \\
\hline & & $J$ & $H$ & $K_{s}$ & {$[3.6]$} & {$[4.5]$} & {$[5.8]$} & {$[8.0]$} & {$[24]$} & \\
\hline 1 & SSTGLMC G048.7567-00.6341 & 15.89 & 14.81 & 14.00 & 12.58 & 12.15 & 11.85 & 10.94 & 7.43 & $\mathrm{~F}$ \\
\hline 2 & SSTGLMC G048.7579-00.2797 & 14.17 & 13.37 & 12.94 & 12.40 & 12.30 & 11.66 & & 6.03 & $\mathrm{~F}$ \\
\hline 3 & SSTGLMC G048.7605-00.0388 & $\ldots$ & $\ldots$ & $\ldots$ & 13.05 & 12.28 & 11.07 & 10.02 & 6.24 & W \\
\hline 4 & SSTGLMC G048.7618+00.0627 & $\ldots$ & $\ldots$ & $\ldots$ & 14.33 & 13.20 & 11.68 & 10.94 & 7.62 & $\mathrm{~W}$ \\
\hline 5 & SSTGLMC G048.7637+00.2022 & $\ldots$ & $\ldots$ & $\ldots$ & 13.80 & 12.37 & 11.27 & 10.67 & 6.47 & $\mathrm{~W}$ \\
\hline 6 & SSTGLMC G048.7655+00.1017 & 14.91 & 14.08 & 13.41 & 12.74 & 12.64 & & & 8.44 & $\mathrm{~F}$ \\
\hline 7 & SSTGLMC G048.7667+00.0766 & 15.97 & 14.10 & 12.82 & 11.33 & 10.80 & 10.31 & 9.84 & 7.60 & $\mathrm{~F}$ \\
\hline 8 & SSTGLMC G048.7702-00.1505 & $\ldots$ & $\ldots$ & $\ldots$ & 13.58 & 12.15 & 11.46 & 11.06 & 6.26 & $\mathrm{~W}$ \\
\hline 9 & SSTGLMC G048.7703+00.0786 & . & $\ldots$ & $\ldots$ & 12.41 & 10.88 & 9.67 & 8.85 & 5.81 & $\mathrm{~W}$ \\
\hline 10 & SSTGLMC G048.7720-00.4792 & 16.27 & 14.38 & 12.90 & 10.94 & 10.53 & 10.03 & 9.25 & & $\mathrm{~F}$ \\
\hline 11 & SSTGLMC G048.7726-00.2215 & $\ldots$ & $\ldots$ & 13.20 & 12.06 & 11.74 & 9.94 & $\ldots$ & 2.28 & $\mathrm{~F}$ \\
\hline 12 & SSTGLMC G048.7744+00.1091 & .. & $\ldots$ & 14.87 & 11.70 & 10.47 & 9.72 & 9.13 & 7.20 & $\mathrm{~W}$ \\
\hline 13 & SSTGLMC G048.7750-00.1507 & $\ldots$ & $\ldots$ & & 13.22 & 12.08 & 10.41 & & 5.34 & W \\
\hline 14 & SSTGLMC G048.7772+00.2513 & $\cdots$ & 14.94 & 13.88 & 12.92 & 13.63 & 11.11 & 9.80 & 3.72 & $\mathrm{~W}$ \\
\hline 15 & SSTGLMC G048.7780+00.2275 & $\cdots$ & 15.19 & 14.29 & 13.20 & 12.72 & 12.15 & 11.56 & $\ldots$ & $\mathrm{F}$ \\
\hline
\end{tabular}

Note. - Table 2 is published in its entirety in the electronic edition of the Astrophysical Journal. A portion is shown here for guidance regarding its form and content.

a Association by the foreground extinction $A_{V}-\mathrm{W}$, W51 source; F, foreground source

TABLE 3

Model Parameters FOR YSO CANDIDATES

\begin{tabular}{|c|c|c|c|c|c|c|c|c|c|c|c|c|c|}
\hline \multirow[t]{2}{*}{ No. } & \multirow{2}{*}{$\begin{array}{c}\text { Name } \\
(\mathrm{G} l+b)\end{array}$} & \multicolumn{3}{|c|}{$A_{V}$ (mag) } & \multicolumn{3}{|c|}{$M_{\star}\left(M_{\odot}\right)$} & \multicolumn{3}{|c|}{$L_{t o t}\left(L_{\odot}\right)$} & \multirow{2}{*}{$\begin{array}{l}\text { Evol. } \\
\text { Stage }\end{array}$} & \multirow{2}{*}{$\begin{array}{c}\text { Class }^{\mathrm{c}} \\
\alpha_{I R A C}\end{array}$} & \multirow{2}{*}{$\begin{array}{l}\text { Class }^{\mathrm{d}} \\
\alpha_{2-24}\end{array}$} \\
\hline & & $\mathrm{L}^{\mathrm{a}}$ & $\mathrm{Ave}^{\mathrm{b}}$ & $\mathrm{U}^{\mathrm{a}}$ & $\mathrm{L}^{\mathrm{a}}$ & $\mathrm{Ave}^{\mathrm{b}}$ & $\mathrm{U}^{\mathrm{a}}$ & $\mathrm{L}^{\mathrm{a}}$ & $\mathrm{Ave}^{\mathrm{b}}$ & $\mathrm{U}^{\mathrm{a}}$ & & & \\
\hline 1 & G048.7567-00.6341 & 2.9 & 4.0 & 5.4 & 2.6 & 3.0 & 3.1 & 43 & 74 & 86 & II & II & II \\
\hline 2 & G048.7579-00.2797 & 0.3 & 1.6 & 2.6 & 1.4 & 2.7 & 4.0 & 17 & 33 & 45 & $0 / \mathrm{I}$ & $\ldots$ & $\mathrm{F}$ \\
\hline 3 & G048.7605-00.0388 & 23.1 & 33.3 & 42.8 & 3.3 & 4.1 & 4.8 & 87 & 269 & 403 & II & I & I \\
\hline 4 & G048.7618+00.0627 & 43.5 & 51.8 & 60.0 & 3.3 & 4.0 & 4.7 & 122 & 274 & 383 & II & I & $\mathrm{F}$ \\
\hline 5 & G048.7637+00.2022 & 6.6 & 25.7 & 53.8 & 2.2 & 3.5 & 4.7 & 49 & 155 & 311 & $0 / \mathrm{I}$ & I & I \\
\hline 6 & $\mathrm{G} 048.7655+00.1017$ & 1.6 & 3.0 & 4.3 & 2.8 & 3.2 & 3.8 & 16 & 66 & 122 & $\mathrm{Amb}$ & $\cdots$ & II \\
\hline 7 & G048.7667+00.0766 & 7.1 & 7.5 & 8.1 & 3.3 & 4.0 & 4.5 & 129 & 229 & 327 & II & II & II \\
\hline 8 & G048.7702-00.1505 & 1.2 & 18.8 & 39.6 & 1.8 & 3.9 & 5.7 & 35 & 106 & 188 & $0 / \mathrm{I}$ & $\mathrm{F}$ & I \\
\hline 9 & G048.7703+00.0786 & 46.5 & 52.7 & 60.0 & 5.4 & 6.6 & 7.8 & 658 & 1788 & 2668 & II & I & $\mathrm{F}$ \\
\hline 10 & G048.7720-00.4792 & 6.2 & 9.3 & 11.4 & 3.8 & 4.6 & 5.5 & 175 & 349 & 456 & II & II & $\mathrm{F}$ \\
\hline 11 & G048.7726-00.2215 & 1.1 & 7.9 & 14.7 & 4.8 & 6.0 & 7.2 & 190 & 472 & 753 & $0 / \mathrm{I}$ & $\cdots$ & I \\
\hline 12 & G048.7744+00.1091 & 24.0 & $34.7^{*}$ & 40.1 & 5.4 & $5.8^{*}$ & 6.0 & 1179 & 1464 & 1536 & II & $\mathrm{F}$ & $\mathrm{F}$ \\
\hline 13 & G048.7750-00.1507 & 4.9 & 32.2 & 56.8 & 1.3 & 4.3 & 6.0 & 46 & 663 & 809 & II & . & I \\
\hline 14 & G048.7772+00.2513 & 13.7 & 36.8 & 58.9 & 2.0 & 3.6 & 5.2 & 98 & 238 & 389 & $0 / \mathrm{I}$ & $\mathrm{I}$ & I \\
\hline 15 & G048.7780+00.2275 & 3.7 & 8.2 & 11.8 & 1.4 & 2.6 & 3.7 & 15 & 35 & 43 & $\mathrm{Amb}$ & II & II \\
\hline
\end{tabular}

Note. - Table 3 is published in its entirety in the electronic edition of the Astrophysical Journal. A portion is shown here for guidance regarding its form and content.

a The range of values reported here on the extinctions, masses, and luminosities for each parameter are the lower and upper limit

of $68 \%$ probability for all of the acceptable YSO models.

b Asterisks indicate ranges of $95 \%$ probability.

c YSO class based on spectral slope $\alpha$ using the flux densities detected in all four IRAC bands.

$\mathrm{d}$ YSO class based on spectral slope $\alpha$ using the flux densities available between 2 and $24 \mu \mathrm{m}$.

fitting tool provides the evolutionary stage and physical parameters such as disk mass, disk accretion rate, and stellar temperature of YSOs. The SED fitting tool has been used to study star forming regions in various environments, e.g., M17 (Povich et al. 2009), the Eagle Nebula (Indebetouw et al. 2007), IRAS 18507+0121 (Shepherd et al. 2007), and the Small Magellanic Cloud (Simon et al. 2007).

Table 1 summarizes the number of sources. We reset the photometric uncertainties to a value of $\delta F / F=10 \%$ for all GLIMPSE Catalog sources, and to $15 \%$ for the MIPS $24 \mu \mathrm{m}$ fluxes including systematic errors based on the GLIMPSE documents ${ }^{6}$. These values were adopted instead of the formal errors in the Catalog in order to fit without possible bias caused by underestimating the

6 http://irsa.ipac.caltech.edu/data/SPITZER/GLIMPSE/doc/ flux uncertainties. A total of 70,767 sources for SED fitting was detected in $N_{\text {data }} \geq 4$ of the $8 \mathrm{IR}$ bands in the GLIMPSE Catalog combined with MIPS $24 \mu \mathrm{m}$ fluxes. Our final goal is to find YSOs associated with molecular clouds, so we start by removing stars. The foreground extinction, up to an allowed maximum $A_{V}$ of 30 mag in this study is accounted for with an extinction law derived from GLIMPSE data (Indebetouw et al. 2005) in the process of fitting stellar photosphere SEDs. We calculate a best-fit $\chi^{2}$ normalized by the number of data points between 4 and 8 used in the fit. We consider all sources with $\chi^{2} / N_{\text {data }} \leq 4$ as good fits to reddened stellar photospheres. The total of sources with $N_{\text {data }} \geq 4$ separates into 69,316 well fit sources and 1,451 badly fit sources by this threshold value. In most cases, even highly reddened stars are classified as good fits because the fitting tool includes the extinction law. 
TABLE 4

Number of YSO CANDidates for Stages and Classes

\begin{tabular}{|c|c|c|c|c|c|c|c|c|c|}
\hline \multirow[t]{2}{*}{ Region } & \multirow[t]{2}{*}{ No. } & \multicolumn{4}{|c|}{ Physical } & \multicolumn{4}{|c|}{ Observational } \\
\hline & & Stage 0/1 & 11 & ПII & Amb. & Class 1 & Flat & 11 & ПII \\
\hline \multicolumn{10}{|c|}{ Sources identified as YSOs with the SED fitter } \\
\hline All ${ }^{\mathrm{a}}$ & 737 & 228 & 255 & 5 & 249 & 256 & 243 & 235 & 3 \\
\hline Fore. ${ }^{b}$ & 300 & 100 & 93 & 5 & 102 & 57 & 75 & 165 & 3 \\
\hline W51 c & 437 & 128 & 162 & 0 & 147 & 199 & 168 & 70 & 0 \\
\hline Outer ${ }^{\mathrm{d}}$ & 281 & 64 & 137 & 0 & 80 & 88 & 132 & 61 & 0 \\
\hline W51A e & 72 & 31 & 9 & 0 & 32 & 52 & 16 & 4 & 0 \\
\hline W51B ${ }^{\mathrm{f}}$ & 84 & 33 & 16 & 0 & 35 & 59 & 20 & 5 & 0 \\
\hline \multicolumn{10}{|c|}{ Sources detected in all four IRAC bands and MIPS $24 \mu \mathrm{m}$} \\
\hline All & 305 & 61 & 196 & 4 & 44 & 85 & 108 & 112 & 0 \\
\hline Fore. & 109 & 26 & 61 & 4 & 18 & 17 & 23 & 69 & 0 \\
\hline W51 & 196 & 35 & 135 & 0 & 26 & 68 & 85 & 43 & 0 \\
\hline Outer & 164 & 24 & 118 & 0 & 22 & 45 & 78 & 41 & 0 \\
\hline W51A & 15 & 8 & 6 & 0 & 1 & 12 & 2 & 1 & 0 \\
\hline W51B & 17 & 3 & 11 & 0 & 3 & 11 & 5 & 1 & 0 \\
\hline \multicolumn{10}{|c|}{ Sources classified as YSOc by Harvey et al. (2007) criteria } \\
\hline All & 270 & 59 & 169 & 1 & 41 & 83 & 97 & 90 & 0 \\
\hline Fore. & 101 & 26 & 57 & 1 & 17 & 17 & 23 & 61 & 0 \\
\hline W51 & 169 & 33 & 112 & 0 & 24 & 66 & 74 & 29 & 0 \\
\hline Outer & 137 & 22 & 95 & 0 & 20 & 43 & 67 & 27 & 0 \\
\hline W51A & 15 & 8 & 6 & 0 & 1 & 12 & 2 & 1 & 0 \\
\hline W51B & 17 & 3 & 11 & 0 & 3 & 11 & 5 & 1 & 0 \\
\hline
\end{tabular}

Entire sample of YSOs identified by SED fitter

b Foreground YSOs with $A_{V}<10 \mathrm{mag}$

c W51 YSOs with $A_{V} \geq 10 \mathrm{mag}$

d YSOs with $A_{V} \geq 10 \mathrm{mag}$ and located in the outside region excluding the central region $\left(48.80^{\circ} \leq l \leq 49.65^{\circ}\right.$ and $\left.-0.48^{\circ} \leq b \leq-0.15^{\circ}\right)$

e YSOs with $A_{V} \geq 10 \mathrm{mag}$ around W51A $\left(49.30^{\circ} \leq l \leq 49.65^{\circ}\right.$ and $-0.48^{\circ} \leq b \leq$ $\left.-0.15^{\circ}\right)$

${ }^{\mathrm{f}}$ YSOs with $A_{V} \geq 10 \mathrm{mag}$ around W51B $\left(48.80^{\circ} \leq l \leq 49.30^{\circ}\right.$ and $\left.-0.48^{\circ} \leq b \leq-0.15^{\circ}\right)$

Next, we fit 1,451 sources that are poorly fit by reddened stellar photospheres using the YSO models of Robitaille et al. (2006). We allow the distance range to be from 5 to $9 \mathrm{kpc}$ and the interstellar extinction from 0 to 60 mag in $V$ band for fitting YSOs. In the same manner as fitting stellar photospheres, we consider sources with $\chi^{2} / N_{\text {data }} \leq 4$ to be well-fit. This YSO fitting process separates these 1451 sources into 1327 good fit YSO sources and 124 bad fit sources. Among good fit sources we discard those not detected at $24 \mu \mathrm{m}$ but which show 8 $\mu \mathrm{m}$ flux excesses above an extrapolation of the 3 shorter wavelength IRAC bands. These sources with $8 \mu \mathrm{m}$ excess emission are usually found to be a noise peak or a diffuse background feature. In cases which show an IR excess in the MIPS $24 \mu \mathrm{m}$ band only, we also discard the source, because these may be a false match due to the difference in resolution between GLIMPSE and MIPSGAL. We extract 14 bright sources which may be evolved stars on the asymptotic giant branch (AGB). For example, carbon stars enshrouded by dusty envelopes have SEDs similar to YSOs. Most of the 124 objects classified as bad fits $\left(\chi^{2} / N_{\text {data }} \geq 4\right)$ are stars with saturated fluxes or signs of variability or bad photometry. After replacing the uncertainties of sources with questionable fluxes to upper or lower limits, we moved five YSO candidates among bad fit sources to the "well-fit" sample. Two samples were moved to the AGB candidate category. After all these adjustments, we distinguish 737 good YSO candidates, 576 bad samples, and 16 bright sources which are likely to be AGB stars.

We divide the final 737 YSO candidates into three evolutionary stages defined by the well-fit model disk mass $M_{\text {disk }}$ and the envelope accretion rate $\dot{M}_{\text {env }}$, both normalized by the mass of the central star $M_{\star}$; Stage 0/I with $\dot{M}_{\text {env }} / M_{\star}>10^{-6} \mathrm{yr}^{-1}$, Stage II YSOs with $\dot{M}_{\text {env }} / M_{\star}<10^{-6} \mathrm{yr}^{-1}$ and $M_{\text {disk }} / M_{\star}>10^{-6}$, and Stage III with $\dot{M}_{\text {env }} / M_{\star}<10^{-6} \mathrm{yr}^{-1}$ and $M_{\text {disk }} / M_{\star}<10^{-6}$. Stage 0/I, II, and III YSOs have significant infalling envelopes, optically thick disks, and optically thin disks, respectively (Robitaille et al. 2006). We determine the evolutionary stage of each source using the relative probability distribution for the stages of all the "well-fit" models. The well-fit models of each source are defined by

$$
\chi^{2}-\chi_{\min }^{2} \leq 2 N_{\text {data }},
$$

where $\chi_{\min }^{2}$ is the goodness-of-fit parameter for the bestfit model. The relative probability of each well-fit model is estimated according to

$$
P\left(\chi^{2}\right)=e^{-\left(\chi^{2}-\chi_{\min }^{2}\right) / 2}
$$

and is normalized. After a probability distribution for the evolutionary stage of each source is constructed from the Stages of all the well-fit models, the most probable stage of each source is determined by the condition that $\Sigma P$ (Stage) $\geq 0.67$. If this condition is not satisfied, then the stage of the source is classified as ambiguous, though the object is still counted as an YSO.

From well-fit models for each source derived from the SED fitting tool, we calculated the $\chi^{2}$-weighted model parameters: interstellar extinction to the source $\left(A_{V}\right)$, stellar mass $\left(M_{\star}\right)$, and total luminosity $\left(L_{t o t}\right.$ : total bolometric luminosity, including luminosity produced by accretion). Note that $A_{V}$ is the foreground interstellar extinction, which does not include the extinction produced by a circumstellar disk or envelope. The $\chi^{2}$ - 

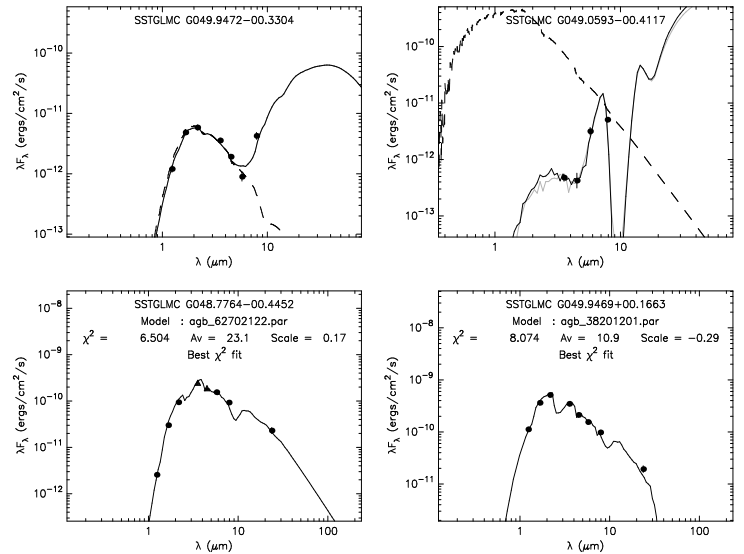

FIG. 3.- Top: Example plots of rejected sources from final samples. Left: Source with IR excess emission only in the IRAC $8.0 \mu \mathrm{m}$ band, but not detected in the MIPS $24 \mu \mathrm{m}$ band. Right: Source with contamination by diffuse background emission in $5.8 \mu \mathrm{m}$ and $8.0 \mu \mathrm{m}$ bands. Bottom: Example SEDs of AGB candidates. Triangles are datapoints with saturated fluxes that were used as lower limits for the model fitting.

weighted average $A_{V}$ distribution for all YSO candidates shows the linear variation in the direction of increasing extinction. We consider 437 YSOs with more than $10 \mathrm{mag} A_{V}$ of foreground extinction as sources associated with the W51 complex because the foreground extinction toward the W51 complex is $A_{V} \sim 20 \mathrm{mag}$ (Goldader \& Wvnn-Williams 1994). The majority (97\% $(192 / 198))$ of the YSO candidates detected in all 2MASS bands have $A_{V}$ values $<10 \mathrm{mag}$, and are classified as foreground objects; while $95 \%(295 / 312)$ of the YSO candidates detected only in IRAC/MIPS bands have fitted $A_{V} \geq 10 \mathrm{mag}$ and are classified as associated with the W51 region. We list magnitudes and classifications for the entire list of 737 YSO candidates in Table 2 . Foreground sources and W51 sources are denoted as "F" and "W", respectively. We present some of the derived physical parameters from the SED fitting for all 737 YSOs in Table 3. We report lower and upper ranges of $68 \%$ probability for each candidate YSO. In cases where the range in the $68 \%$ probability distribution is very small, we list ranges for the $95 \%$ confidence intervals and mark these with an asterisk.

In the last three columns of Table 3, we give evolutionary stages and SED classes for each YSO candidates. We classify the 737 YSO candidates as 228 Stage 0/I, 255 Stage II, 5 Stage III, and 249 ambiguous YSO candidates based on the disk mass $M_{\text {disk }}$ and the envelope accretion rate $\dot{M}_{\text {env }}$ of each source. The spectral index $\alpha_{I R A C}$ is the value derived from the flux densities of the sources detected in all four IRAC bands. Among 737 YSO candidates 561 sources are detected in all four IRAC bands and divided into 83 Class I, 164 Flat, 195 Class II, and 19 Class III. Using the flux densities available between 2 and $24 \mu \mathrm{m}$ to derive the spectral index, $\alpha_{2-24}$, all sources are classified as 256 Class I, 243 Flat, and 235 Class II, and 3 Class III. We summarize the physical Stages and observational Classes of YSOs in Table 4.

Figure 2 $(d)$ shows the distribution of 561 YSO candidates detected in all 4 IRAC bands in the IRAC [3.6] - [4.5] vs. [5.8] - [8.0] color-color diagram for comparison with Figure $2(b)$ and $(c)$. A total of 176 YSO candidates ( $24 \%$ of the total YSOs) are identified from a combination of $\mathrm{JHK}_{\mathrm{s}}$ and MIPS $24 \mu \mathrm{m}$ fluxes although these are not detected in all 4 IRAC bands. In Figure 2(d), YSO candidates are classified according to their most probable evolutionary Stage: red for Stage 0/I, yellow for Stage II, and cyan for Stage III. Since Stage III YSOs with optically thin remnant disks and their SEDs are dominated by photospheric emission, those objects are difficult to identify by IR excess alone. Despite a low probability, 5 Stage III sources are in our final samples because the evolutionary Stage is determined from multiple YSO models of each YSO candidate statistically. Green dots are for ambiguous sources that are YSO candidates but do not have a sufficiently well-defined probability distribution to determine the evolutionary Stage. Blue dots are sources that were well-fit by YSO SEDs but were not included in the final sample. Figure 3, top shows examples of these rejected sources with IR excess emission only in the IRAC $8.0 \mu \mathrm{m}$ band (left) and with contamination by diffuse background emission (right). Examples of SEDs for two AGB candidates are plotted in Figure 3, bottom.

\subsection{Estimating Contamination}

Because other IR-excess populations (e.g., AGB stars and galaxies) have colors or SEDs very similar to YSOs, the SED-fitting method will inevitably have contamination from these sources. Therefore we use an empirical method to estimate the contamination of YSO candidates from galaxies and dusty evolved AGB stars. GLIMPSE is a shallow survey of the Galactic plane while c2d is a deep survey of nearby star forming regions away from the Galactic plane: Chameleon II, Lupus, Perseus, Serpens, and Ophiuchus (Evans et al. 2009). The contamination by external galaxies is serious in deep IRAC observations, such as the c2d Survey (Harvey et al. 2007; (Porras et al. 2007). In GLIMPSE, evolved stars like AGB stars with dusty winds and unresolved planetary nebulae $(\mathrm{PNe})$ may be more serious contamination sources than external galaxies (Povich et al. 2009).

To estimate an upper limit for evolved star (AGB) contamination, we use the color criterion ([8.0] $[24]<2.2 \mathrm{mag}$ ) of Whitney et al. (2008). Their colormagnitude criterion derived from the LMC is not appropriate for W51, given the difference in distance and the fact that the AGB stars will not all be at the same distance as the YSOs. Note that relatively few extreme AGB stars are expected to populate the upper-right region of the [8.0] vs. [8.0] - [24] color-magnitude diagram, and in the Galaxy such sources are likely to be saturated in GLIMPSE. Sources with [8.0] $-[24]<2.2$ mag are 10 of our 345 YSO candidates detected in [8.0] and [24] $\mu \mathrm{m}$ bands, or $\sim 3 \%$ (Figure $4(b)$ ). They do yield a similar (low) surface density of AGB stars as found for the M17 region by Povich et al. (2009). The distribution of red sources in the Galactic midplane (Robitaille et al. 2008), which consist mostly of YSOs and AGB stars, also shows that the number of YSO candidates represents a significant enhancement toward the W51 region while the surface density of candidate AGBs is very low in our region of $1.25^{\circ} \times 1.00^{\circ}$ centered on $(l, b)=\left(49.375^{\circ},-0.2^{\circ}\right)$.

For each source detected in all IRAC bands and in MIPS $24 \mu \mathrm{m}$, we calculated the probability of being an extragalactic contaminant using the formulas in Harvey et al. (2007). These formulas were derived for 

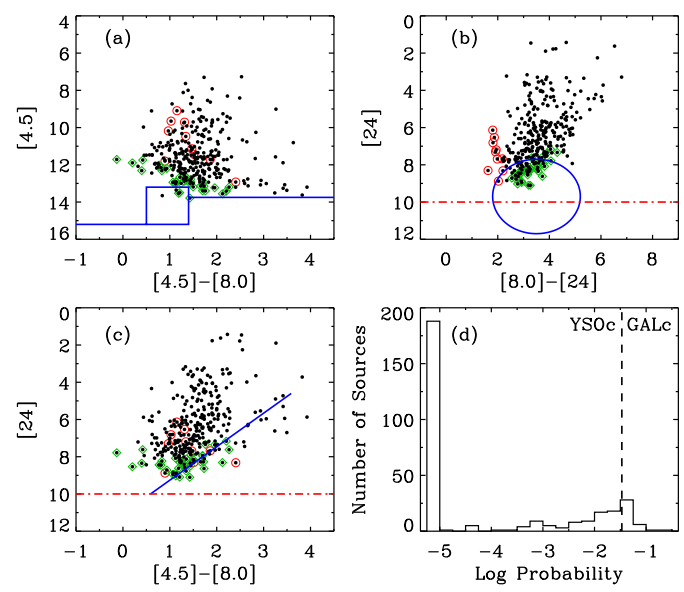

FIG. 4.- $(a),(b),(c)$ Color-magnitude diagrams of YSO candidates in W51. The solid lines show the color-magnitude cuts for defining the YSO candidate criterion developed by Harvey et al. (2007) but with a total extinction of $A_{V}=25 \mathrm{mag}$ along the line of sight, rather than the average value of $7.5 \mathrm{mag}$ for the Serpens cloud. The dot-dashed lines show hard limits, fainter than which objects are excluded from the YSO category. Open symbols marked by circles and diamonds are objects which may be AGBs and extragalactic sources. (d) Plot of the number of sources vs. probability of being a background contaminant. The vertical dashed line shows the separation chosen by Harvev et al. (2007) for YSOs vs. extragalactic candidates.

the Serpens molecular cloud field, which has an average extinction of $A_{V} \sim 7.5 \mathrm{mag}$, from the distribution found by Harvey et al. (2007). The Serpens cloud lies at $b=5^{\circ}$ latitude, so almost all of the extinction must be associated with the cloud. In contrast, our W51 field is right in the plane of the Galaxy so the line of sight to any external galaxy should have a minimum extinction corresponding to at least $25 \mathrm{kpc}$ in the plane. We assume a minimum of $1 \mathrm{mag} / \mathrm{kpc}$ or $A_{V} \geqq 25 \mathrm{mag}$ for our field or 17.5 mag more than used for the Serpens field. We therefore adjust the Harvey et al. (2007) criteria by the expected additional extinction at [4.5], [8.0], and [24], using the extinction laws of Indebetouw et al. (2005) and Flaherty et al. (2007). These give an additional $0.7 \mathrm{mag}$ in all three of the IR bands. We add this amount to the constants for the 3 IR magnitudes in the formulae of Harvev et al. (2007). When we applied this modified set of color-magnitude criteria to 305 YSO candidates detected in all IRAC bands and in MIPS $24 \mu \mathrm{m}, 35$ sources were in the galaxy-candidate region of the probability histogram using the Harvev et al. (2007) cutoff of log P $=-1.47$. We note, however, that this cutoff represents a very conservative estimator, i.e., a probability as low as 0.034 counts as a galaxy candidate. Figure 4 presents three color-magnitude diagrams showing the possibility of contamination using the modified Harvey et al. (2007) criteria, where the solid lines from their formulae are shifted down by $0.7 \mathrm{mag}$ in the magnitude axes. The joint probability histogram in Figure 4( $d$ ) shows that, of the 35 sources in the nominal GALc region, only 8 occupy the bins with a probability greater than 0.06 (log $\mathrm{P}>-1.25)$ of being an extragalactic contaminant, i.e., about $3 \%$ of the sample of 305 objects.

We therefore estimate an upper limit of about $6 \%$ to the contamination by galaxies and AGB stars in our list of YSOs. This limit implies a smaller contamination fraction compared to other regions, e.g., 10\% for M17 (Povich et al. 2009), 40\% for the intrinsically red sources in the Galactic midplane (Robitaille et al. 2008), and $55 \%$ for the LMC (Whitnev et al. 2008). Therefore, considering up to $2 \%$ contamination by unresolved Planetary Nebulae (Robitaille et al. 2008; Whitney et al.|2008), we estimate an upper limit to the contamination of $8 \%$ in our YSO candidates.

\section{RESULTS AND DISCUSSION}

Our target area contains very active star-forming regions in the disk of the Milky Way (Figure 1). We find a total of 737 YSO candidates within the region around the W51 complex based on the SED fitter. After dividing 737 YSOs into foreground and W51 YSOs based on the amount of foreground extinction, we focus on 437 YSO candidates associated with the W51 complex.

These 437 sources divide into 199 Class I, 168 Flat, and 70 Class II when classified by the empirical SED spectral index, $\alpha_{2-24}$. For comparison, the percentage of sources in these observational SED classes for all c2d clouds is reported as $16 \%$ Class I, $12 \%$ Flat, $60 \%$ Class II, and $12 \%$ Class III (Evans et al. 2009). The ratio of Class I plus Flat spectrum to Class II sources in W51 is about 5, which is completely different from the low mass star-forming regions such as Cha II (0.16), Lupus (0.29), Perseus (0.57), Serpens (0.42), and Ophiuchus (0.47) (Evans et al. 2009). The physical "Stages", I, II, and III should roughly correspond to the observational "Classes" I+Flat, II, and III (Harvey et al. 2007). In W51 the 168 Flat sources divide into 30 Stage 0/I, 75 Stage II, and 63 with ambiguous stage. Using the physical evolutionary models for all sources associated with W51, 128 are classified as Stage 0/I, 162 as Stage II, and 147 as ambiguous sources. The massive star-forming region W51 appears to contain YSOs in a very early stage as indicated by both observational and physical classifications. The ratio of Stage 0/I to II for the entire set of YSOs in the large $1.25^{\circ} \times 1.00^{\circ}$ region is similar to that for other massive star forming regions like M17 (25/40; Povich et al. 2009), N66 in the SMC (33/50; Simon et al. 2007), and the LMC (145/147; Whitney et al. 2008).

In the central active star-forming region (inside the boxed area in Figure 7) the ratio of Stage 0/I to II for all YSOs is $2.56(64 / 25)$. In the same region this ratio for the massive YSOs $\left(>5 M_{\odot}\right)$ only is $2.71(46 / 17)$. The ratio of YSOs outside the central bright region is $0.47(64 / 137)$ and the ratio for sources greater than $5 M_{\odot}$ in the same region is $0.92(23 / 25)$. The increase in Stage $0 / \mathrm{I}$ to II is due in part to a bias toward detecting sources with positive spectral indices in regions of bright mid-IR nebular emission. M17 provides an extreme example of bright mid-IR nebular emission, where the ratio reaches 1.3 $(13 / 10)$ in the central bright region (Povich et al. 2009). In both W51 and M17, the relative fraction of sources with Ambiguous Stage determinations increases in the bright nebular regions, due to the decreased sensitivity of the MIPS $24 \mu \mathrm{m}$ point-source detections, an important discriminant between Stage 0/I and Stage II sources (Indebetouw et al. 2007). However, these selection biases become less important for more luminous sources, and the large number of Stage 0/I objects with $M>5 M_{\odot}$ found in our sample, 69 , is unprecedented among studies of Galactic star formation regions to date. It implies that 

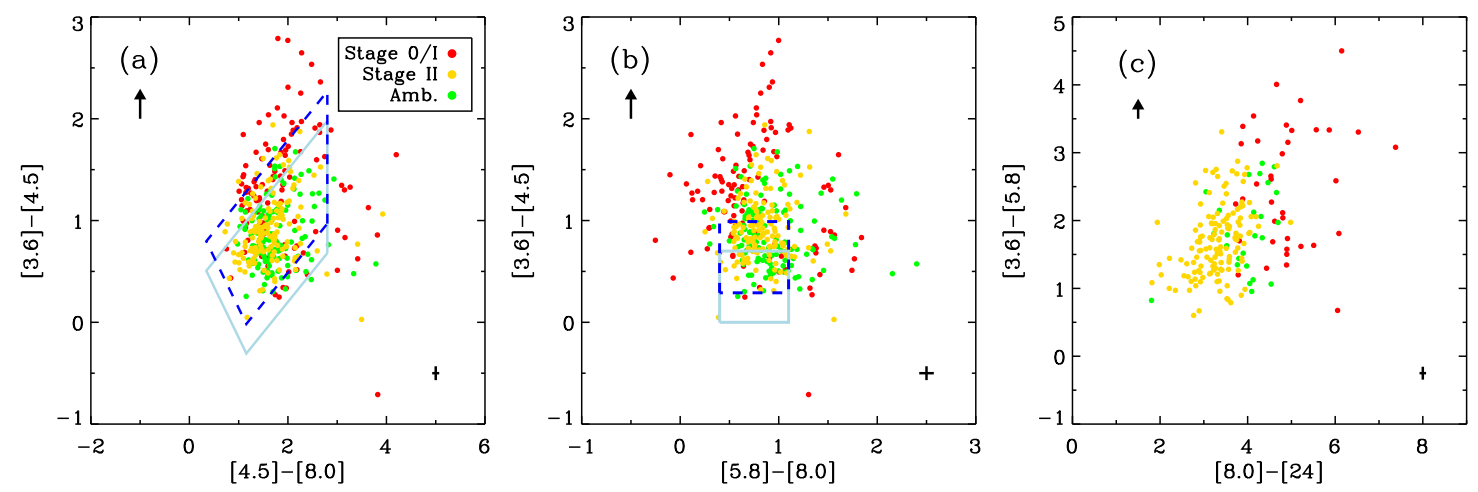

FIG. 5.- (a) IRAC [3.6] - [4.5] vs. [4.5] - [8.0] color-color diagram with YSO candidates selected by the SED fitting tool. Points in red mark Stage 0/I, yellow mark Stage II, and green mark ambiguous sources. The solid line represents the region of the color selection criteria of Simon et al. (2007). The dashed line shows the region shifted by the reddening vector for the general visual extinction toward W51 of 20 mag (Goldader \& Wynn-Williams 1994). (b) IRAC [3.6] - [4.5] vs. [5.8] - [8.0] color-color diagram. The solid line represents the disk domain of Allen et al. (2004). The dashed line shows the region shifted by the reddening vector. (c) [3.6] - [5.8] vs. [8.0] - [24] color-color diagram. Reddening vector for $\mathrm{A}_{V}=20 \mathrm{mag}$ based on the extinction laws of Indebetouw et al. (2005) and Stutz et al. (2009, in preparation) is shown as an arrow. Black crosses show typical photometric errors.

W51 is not only very massive but that it could also be exceptionally young, even when compared to M17. This result is consistent with the idea that W51 IRS 2E, the most luminous embedded source in W51A, is a highly embedded, very young $\mathrm{O}$ star that has yet to produce a detectable hypercompact H II region (Figuerêdo et al. 2008). ${ }^{7}$

\subsection{Color-Color Diagrams}

Figure 5 shows the distribution of the final 437 YSO candidates in IRAC color-color diagrams. The sources plotted in Figure 5]are classified according to their most probable evolutionary Stage from the Robitaille et al. (2006) models. Red dots are Stage 0/I YSOs that are relatively unevolved, heavily embedded in their natal envelopes. Yellow dots are Stage II YSOs, like Class II $\mathrm{T}$ Tauri stars, with optically thick circumstellar disks. There are no Stage III YSOs with optically thin remnant disks in this sample. Green dots are objects with ambiguous stage classification. In Figure [5( $a)$, we see that the color selection criteria of Simon et al. (2007) includes many reddened stars as well as YSO candidates within the solid line. If the color selection criteria are shifted by $\sim 0.3 \mathrm{mag}$ in the [3.6] $-[4.5]$ color (corresponding to $A_{V}=20 \mathrm{mag}$ ), relatively clean YSO candidates are selected inside the dashed line. In Figure $5(b)$, if the "disk domain" defined by Allen et al. (2004) also moves vertically in the [3.6] - [4.5] color, many Stage II YSO candidates with optically thick circumstellar disks fall in the disk domain region. The shift of the color selection criteria in the direction of the reddening vector is consistent with the fact that the W51 complex is a more massive star forming region with more extinction than the star forming region studied by Allen et al. (2004). Figure $5(c)$ shows the importance of the $24 \mu \mathrm{m}$ flux in classifying the evolutionary stages of YSO candidates. Stage 0/I objects (red dots) are redder than Stage II objects (yellow dots) in [8.0] - [24] color space. Most of the ambiguous sources (green dots) are in the boundary region between Stage 0/I and Stage II.

7 IRS $2 \mathrm{E}$ is saturated in the GLIMPSE and MIPSGAL images, hence it is not included in our sample of candidate YSOs.

\subsection{Spatial Distribution of $Y S O s$}

Figure 6] shows the spatial distribution, classified by evolutionary stage, of the 437 YSO candidates associated with the W51 region on the IRAC $8 \mu \mathrm{m}$ image. YSO candidates are marked in red for Stage 0/I, yellow for Stage II, and green for ambiguous sources by evolutionary stage. Stage 0/I YSOs are clearly concentrated toward the bright H II regions. Stage II YSOs are distributed outside of the central dense region. Most sources within the bright $8 \mu \mathrm{m}$ emission near the central region show strong clustering. These clustered sources are associated with the W51 molecular cloud. Several clusters of YSOs are also apparent in the outer region. A very strong concentration of YSOs is found in an IR dark cloud (IRDC) centered on $(l, b)=\left(49.4^{\circ}, 0.0^{\circ}\right)$. The detailed analysis of the $\mathrm{CO}$ observations and IRDC associated with this YSO clustering will be presented elsewhere (Kang et al., in preparation).

Figure 7 shows the spatial distribution of the YSOs classified by the mass of the central star on the IRAC 8 $\mu \mathrm{m}$ image. The vicinity of the compact radio continuum sources marked as crosses in Figure 1 is bright in the IRAC $8 \mu \mathrm{m}$ band due to PAH emission features stimulated by UV radiation from $\mathrm{H}$ II regions. The mass range of YSOs is from 1 to $18 M_{\odot}$. We divide all YSOs into three mass ranges: $M_{\star} \leq 5 M_{\odot}, 5 M_{\odot} \leq M_{\star}<8 M_{\odot}$, $M_{\star} \geq 8 M_{\odot}$. Most of the massive YSOs $\left(\geq 8 M_{\odot}\right)$ are located near the main H II regions of W51. Clustering of massive YSOs is clearly apparent in the central region, while the YSO cluster centered on $(l, b)=\left(49.4^{\circ}\right.$, $\left.0.0^{\circ}\right)$ consists of many lower mass YSOs and only one massive YSO. The mass distribution of YSOs shows evidence of mass segregation with the most massive objects exhibiting a strong concentration close to the center of the H II regions. We can quantify this trend by computing the mass function of YSOs for various regions within our target area.

\subsection{Mass Function of $Y S O s$}

We investigate the variation with spatial distribution of the mass function (MF) of YSOs. We divide our target field $\left(1.0^{\circ} \times 1.25^{\circ}\right)$ into two regions: the central part including very bright emission in all bands from near-IR 


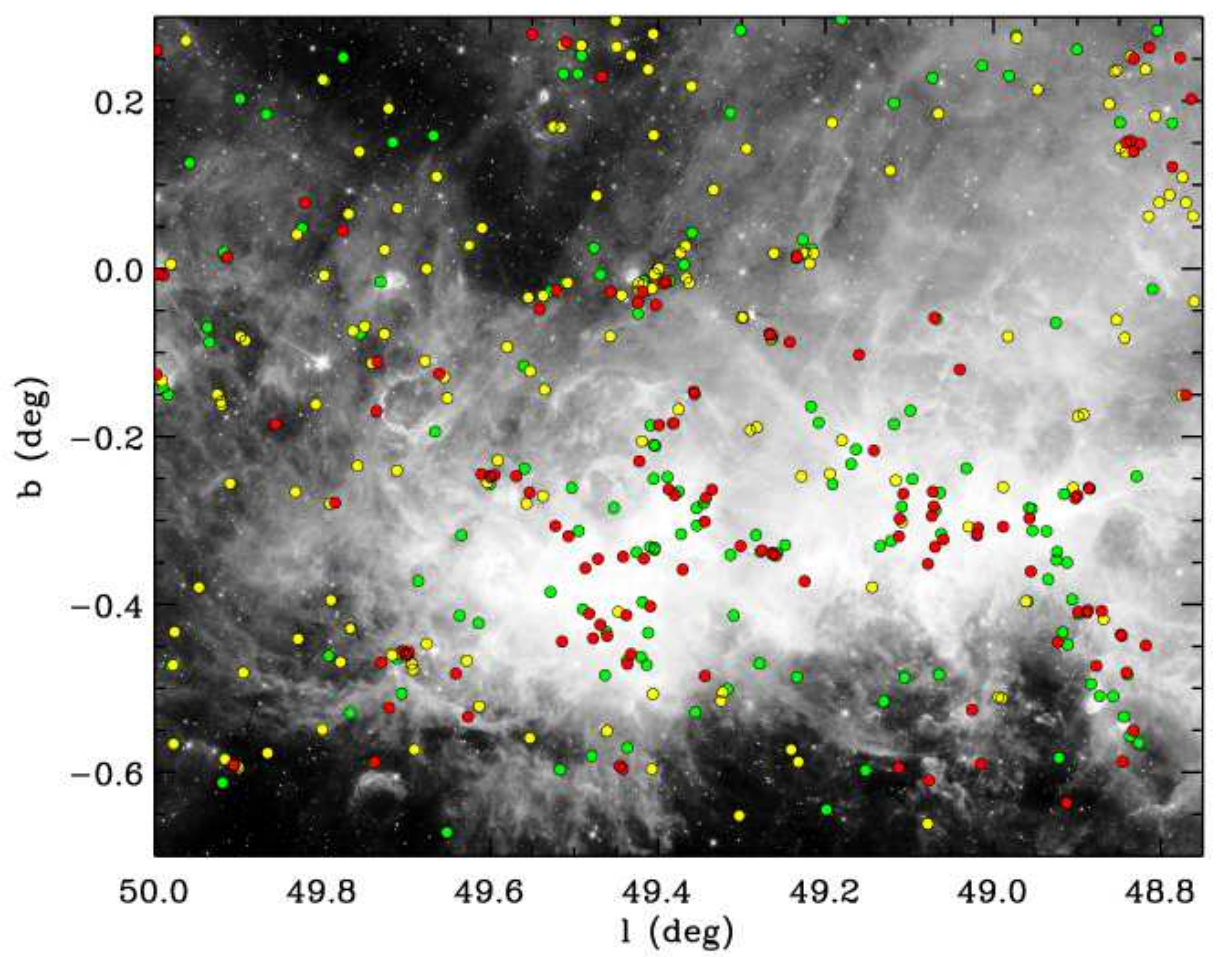

FIG. 6. - Spatial distribution of YSOs in W51, overplotted on IRAC $8.0 \mu \mathrm{m}$ image. YSO candidates are marked in red for Stage 0/I, yellow for Stage II, and green for ambiguous sources.

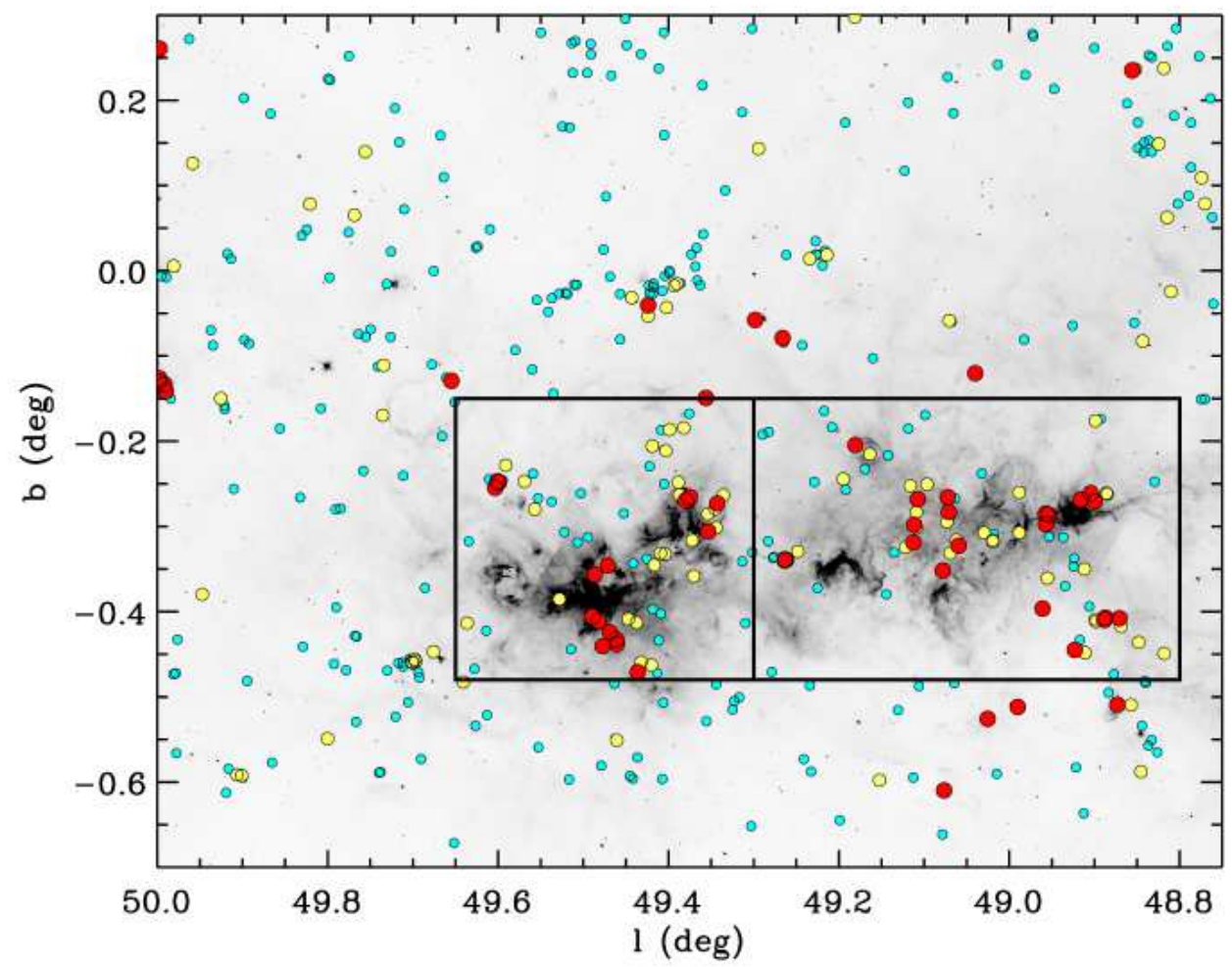

FIG. 7. - The mass distribution of 437 YSOs associated with the W51 region overplotted on the IRAC $8.0 \mu \mathrm{m}$ image (This is the same image as in Figure 6 but displayed with inverted grayscale image to show more clearly the structure of very strong PAH emission in black). Small (cyan), medium (yellow) and large (red) dots represent YSOs with $M \leq 5 M_{\odot}, 5 M_{\odot} \leq M<8 M_{\odot}$ and $M>8 M_{\odot}$. Box shows the boundary of the central region for comparing the shape of the IMF. Left side of the box is the W51A region and right side is the W51B region. 


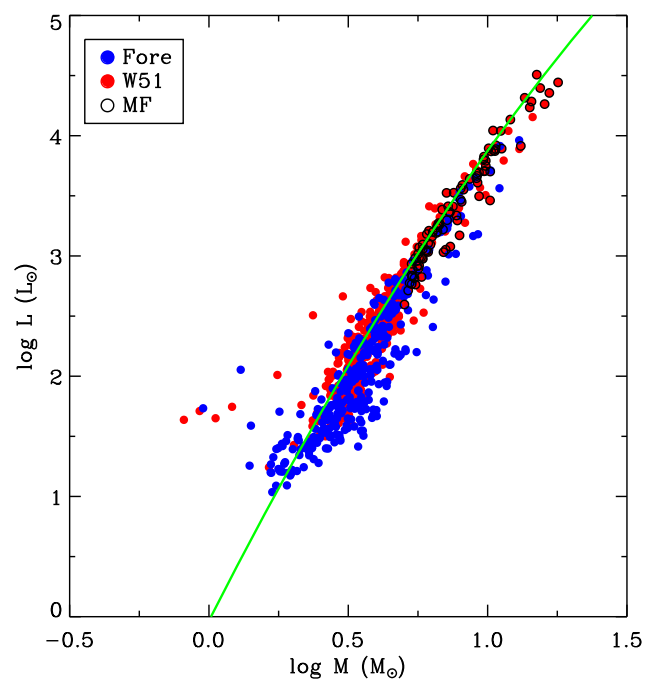

FIG. 8.- The mass-luminosity relation for YSOs in the W51 region. Blue and red dots represent foreground and W51 sources, respectively. Open circles are the sources used for MF derivation. Green line shows the ZAMS mass-luminosity relation by Malkov (2007) for intermediate mass stars $\left(1-30 M_{\odot}\right)$.

to $21 \mathrm{~cm}$ continuum $\left(48.80^{\circ} \leq l \leq 49.65^{\circ}\right.$ and $-0.48^{\circ} \leq$ $b \leq-0.15^{\circ}$ shown by the solid box in Figure 7) and the outside region excluding the central region. We also find the MF of the W51A and W51B regions of the central part, separately.

To obtain as clean a sample as possible, we remove from the final source list those objects with a probability of being extragalactic and AGB contaminants, as discussed in Section 2.4. To compare MFs without bias of source selection we set two criteria. The sensitivity limit of IRAC sources is affected by the brightness of the diffuse emission, in particular at IRAC $8.0 \mu \mathrm{m}$. Therefore, the sensitivity limit in star-forming regions with bright diffuse emission is certainly different from the outside region. Robitaille et al. (2008) found that sources with lower limits on the IRAC 4.5 and $8.0 \mu \mathrm{m}$ fluxes of 0.5 and $10 \mathrm{mJy}$ respectively are not affected by the variations in the diffuse emission on a Galaxy-wide scale. We apply these lower limits on the IRAC 4.5 and $8.0 \mu \mathrm{m}$ fluxes to our final YSO sources, which selects 113 YSOs. The mass distribution of 113 YSOs turns over at $5 M_{\odot}$. Therefore we use only those 94 YSOs with $M_{\star}>5 M_{\odot}$ for comparing the shape of the mass function of YSOs in the W51 region to avoid introducing bias due to sensitivity and completeness. Although we have used very conservative brightness cutoffs in constructing our mass functions, the mass functions are unlikely to be $100 \%$ complete in the central regions, due to the selection effects imposed by confusion and extremely bright nebular emission. In the case of more luminous objects, the stellar luminosity dominates in the total luminosity of the source, while the accretion luminosity is clearly negligible, so the SED-determined luminosity simply sets the corresponding stellar mass given the distance. We find that the mass-luminosity relation for the sources used in the MF derivation agrees with a recent re-evaluation of the ZAMS mass-luminosity relation by Malkov (2007), as shown in Figure 8 . The mean distance of 94 YSOs derived from the SED fitter is $6.6 \pm 0.2 \mathrm{kpc}$ which is close to the $6.1 \pm 1.3 \mathrm{kpc}$ measured for water masers in the W51A region (Imai et al. 2002).

The mass distribution from 94 YSOs which satisfy the sensitivity and completeness criteria in our target region has a best-fit slope of the MF, $\Gamma=-1.70 \pm 0.10$, as defined by

$$
M_{\star}^{\Gamma} \propto \frac{d \xi\left(M_{\star}\right)}{d\left(\log M_{\star}\right)}
$$

for $M_{\star}>5 M_{\odot}$ (solid line in Figure $\left.9(a)\right)$. This value is very close to the stellar IMF slope of $\Gamma=-1.8$, derived by Rana (1987) for the solar neighborhood field stars $\left(1.5 M_{\odot}-100 M_{\odot}\right)$. Foreground sources which satisfy the same sensitivity and completeness have the MF, $\Gamma=-2.13 \pm 0.29$ (dash-dotted line in Figure 9 $(a)$ ). However the YSO MFs of the central active star forming region $(\Gamma=-1.26 \pm 0.12)$ and the outer region $(\Gamma=-2.36 \pm 0.26)$ are significantly different in Figure 9 (b). The MF for the inner region has a slope similar to the Salpeter law, while the MF of the outer part is very steep. Kroupa \& Weidner (2003) note that field-star IMFs are always steeper for masses over $1 M_{\odot}$ than the stellar IMF derived from an individual massive star cluster. In the Large and Small Magellanic Clouds, Massev (2002) found a very steep IMF slope $(\Gamma \sim-4 \pm 0.5)$ outside of the OB associations. The IMF is often observed to be shallower in the central regions of clusters. This mass segregation effect might occur because the most massive stars are either born near the center or migrate toward the center. The question is then whether this segregation is due to initial conditions or to dynamical evolutionary effects. In the W51 H II region complex, most of the massive YSOs in the early stages of evolution are concentrated in the central region (see Figure 6 and (7). Because we derive the MFs using YSOs in the early stages, still surrounded by disks and/or infalling envelopes, these YSOs must be located in their birthplaces. Therefore the spatial variation of the MF in W51 indicates that the mass segregation is caused by initial conditions, not dynamical effects. The difference between the central and outer regions is whether the sources are associated with the most active star-forming region or not. In the outer region YSOs may be forming in relatively small or isolated molecular clouds.

We compare MF of YSOs calculated from Spitzer observation to the stellar mass function. IMFs of many dense clusters in the solar neighborhood are close to the Salpeter IMF slope, $\Gamma=-1.35$ on a plot with logmass intervals. Scalo (1986, 1998) reviewed the determination of the stellar IMF extensively and concluded the IMF slope of field stars to be $-1.7 \pm 0.5$ in the range of $2-10 M_{\odot}$. Ninkov et al. (1995) found a slope of $\Gamma=-1.38 \pm 0.19$ for masses between $2.5-30 M_{\odot}$ from the $\mathrm{OB}$ cluster in the $\mathrm{H}$ II region IC 1805 in the Cas OB6 association associated with the molecular cloud W4. They noted that the mass function determined from young galactic OB clusters varies from $\Gamma=-1.0$ to -1.4 . Garmany et al. (1982) determined a slope of the IMF of $\Gamma=-1.6$ for massive stars $\left(>20 M_{\odot}\right)$ within $2 \mathrm{kpc}$ of the Sun. They found evidence that the slope of the IMF varied with galactic radius: the IMF of stars inside the solar circle is $\Gamma=-1.3$ and outside is $\Gamma=-2.1$. They suggested that the excess of massive stars inside the solar circle was due to the $\mathrm{OB}$ associations in the 


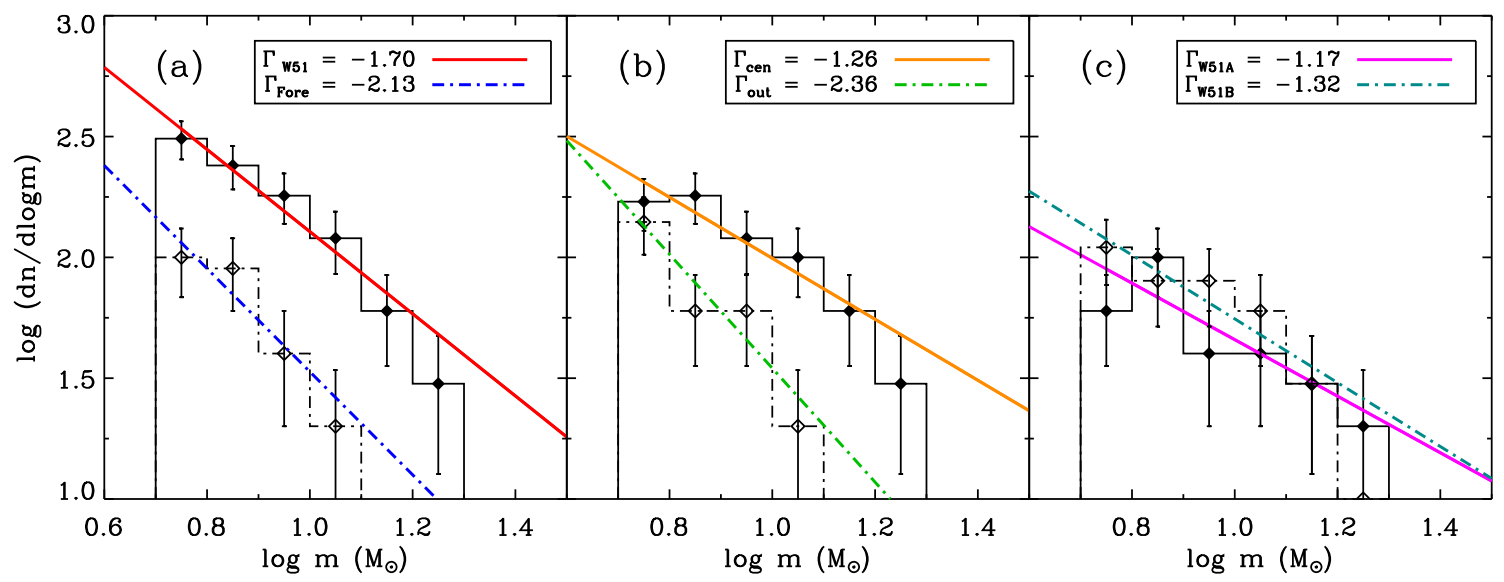

FIG. 9. - (a) The mass function for YSO candidates after selecting only $>0.5$ and $10 \mathrm{mJy}$ at 4.5 and $8.0 \mu \mathrm{m}$ and cut off $>5 M_{\odot}$. The solid line and the dash-dotted line represent the mass function of YSOs associated with W51 and foreground YSOs, respectively. (b) The mass function of the central region (box in Figure 7) (solid line) and the outside region (dash-dotted line). (c) The mass function of the W51A (solid line) and the W51B (dash-dotted line) region. All histograms are shown with $\sqrt{N}$ errorbars.

Carina and Cygnus spiral arms. Hunter et al. (1996) estimated an IMF slope of $-1.6 \pm 0.7$ for intermediate-mass stars $\left(6.5-18 M_{\odot}\right)$ from NGC 604 in the galaxy M33. Massey \& Hunter (1998) derived a slope close to the Salpeter value from the 30 Doradus region of the LMC, suggesting that there is no strong dependence of the IMF slope on metallicity. Sabbi et al. (2008) found a presentday mass function of $\Gamma=-1.43 \pm 0.18$ of NGC 346, the active star-forming region in the SMC. Homeier \& Alves $(2005)$ derived a slope of $\Gamma=-1.6 \pm 0.3$ in the giant radio H II region W49A. Okumura et al. (2000) found that the IMF of the massive stars $\left(M \geq 4 M_{\odot}\right)$ in $\mathrm{G} 49.5-0.4$ of W51A is consistent with an IMF slope of -1.8 , but that the IMF shows a statistically significant excess of stars in the high mass range above $30 M_{\odot}$. These various observations suggest that low density regions have slightly steeper IMFs than the Salpeter slope, while more massive clusters have a more massive upper end of the IMF (Elmegreen 2004, 2006). The results we present here appear to support this conclusion.

We divide the central active star-forming region into W51A and W51B (Figure 7) and find the slopes of the $\mathrm{MF}$ of $\Gamma=-1.17 \pm 0.26$ and $\Gamma=-1.32 \pm 0.26$, respectively (Figure $9(c)$ ). The slopes are the same as the Salpeter IMF within the errors. Similar results that the MFs of YSOs are consistent with the Salpeter IMF are reported from other active star-forming regions, e.g., M17 in the Galaxy (Povich et al. 2009), N66 in the SMC (Simon et al. 2007), and the LMC (Whitney et al. 2008). It is not surprising that our MF of YSOs in this active star-forming region is similar to the stellar IMF because even the core mass function, which has $\Gamma=-1.3$ for $M>0.8 M_{\odot}$ in Perseus, Serpens, and Ophiuchus, is consistent with the Salpeter IMF (Enoch et al. 2008). In a future paper, we will present the detailed properties of molecular clouds associated with the YSOs in W51A and W51B using our ${ }^{12} \mathrm{CO}$ and ${ }^{13} \mathrm{CO} J=2-1$ emission line maps of the region studied in the present work.

\section{SUMMARY}

We have found 737 candidate YSOs in a $1.25^{\circ} \times 1.00^{\circ}$ area near the W51 GMC using Spitzer Space Telescope data. We distinguish YSOs from main-sequence or red gi- ant stars, asymptotic giant branch stars, and background galaxies by fitting model SEDs to fluxes of sources. We divide the total of 737 YSOs into two groups based on interstellar extinction for each YSO: sources associated with the W51 region and foreground sources. We identify 69 highly embedded Stage 0/I candidate YSOs in our field with masses $>5 M_{\odot}$ (corresponding to mid- to early-B main-sequence spectral types) 46 of which are located in the central active star forming regions of W51A and W51B. From the YSOs associated with W51, we find evidence for mass segregation showing that the most massive YSOs are concentrated on the W51 H II region complex. We find a variation in the spatial distribution of the MF of YSOs in the mass range between $5 M_{\odot}$ and 18 $M_{\odot}$. The mass distribution from YSOs in our entire target region has a slope of $\Gamma=-1.70 \pm 0.10$. When the sample is divided into the active star-forming region and the surrounding outer region, the slope of MFs in the starforming region $(\Gamma=-1.26 \pm 0.12)$ is shallower than that of outer region $(\Gamma=-2.36 \pm 0.26)$. However, we do not find any difference in slopes between two active star forming regions within the complex: $\Gamma=-1.17 \pm 0.26$ and $\Gamma=-1.32 \pm 0.26$ in W51A and W51B, respectively. The variation of the MF for YSOs embedded in the molecular cloud implies that the distribution of stellar masses in clusters depends on the local conditions in the parent molecular cloud.

We thank Barbara A. Whitney for a helpful discussion in the very early stage of this work. We thank Brian L. Babler and Marilyn R. Meade for help with MIPS photometry and Bon-Chul Koo for providing 21 $\mathrm{cm}$ radio continuum data. The authors also thank the anonymous referee for helpful comments that improved the text. M. K. also thanks Yujin Yang and Amy Stutz for helpful discussion. This research was supported in part by NSF grant AST-0708131 to the University of Arizona. This work was supported by the Korea Research Foundation Grant funded by the Korean Government (MOEHRD: KRF-2007-612C00050). MSP is supported by an NSF Astronomy and Astrophysics Postdoctoral Fellowship under award AST-0901646. 


\section{REFERENCES}

Allen, L. E., et al. 2004, ApJS, 154, 363

Benjamin, R. A., et al. 2003, PASP, 115, 953

Benjamin, R. A., et al. 2005, ApJ, 630, L149

Bieging, J. 1975, in "H II regions and related topics", eds. T.L. Wilson \& D. Downes, Lecture Notes in Physics, Vol. 42 (Berlin: Springer), p. 443

Carey, S. J., et al. 2005, Bulletin of the American Astronomical Society, 37, 1252

Carpenter, J. M., \& Sanders, D. B. 1998, AJ, 116, 1856

Elmegreen, B. G. 2004, MNRAS, 354, 367

Elmegreen, B. G. 2006, arXiv:astro-ph/0610687

Enoch, M. L., Evans, N. J., II, Sargent, A. 1., Glenn, J., Rosolowsky, E., \& Myers, P. 2008, ApJ, 684, 1240

Evans, N. J., et al. 2009, ApJS, 181, 321

Fazio, G. G., et al. 2004, ApJS, 154, 10

Figuerêdo, E., Blum, R. D., Damineli, A., Conti, P. S., \& Barbosa, C. L. 2008, AJ, 136, 221

Flaherty, K. M., Pipher, J. L., Megeath, S. T., Winston, E. M., Gutermuth, R. A., Muzerolle, J., Allen, L. E., \& Fazio, G. G. 2007, ApJ, 663, 1069

Garmany, C. D., Conti, P. S., \& Chiosi, C. 1982, ApJ, 263, 777

Goldader, J. D., \& Wynn-Williams, C. G. 1994, ApJ, 433, 164

Greene, T. P., Wilking, B. A., Andre, P., Young, E. T., \& Lada, C. J. 1994, ApJ, 434, 614

Gutermuth, R. A., et al. 2008, ApJ, 674, 336

Harvey, P., Merín, B., Huard, T. L., Rebull, L. M., Chapman, N., Evans, N. J., II, \& Myers, P. C. 2007, ApJ, 663, 1149

Homeier, N. L., \& Alves, J. 2005, A\&A, 430, 481

Hunter, D. A., Baum, W. A., O'Neil, E. J., Jr., \& Lynds, R. 1996, ApJ, 456, 174

Imai, H., Watanabe, T., Omodaka, T., Nishio, M., Kameya, O., Miyaji, T., \& Nakajima, J. 2002, PASJ, 54, 741

Indebetouw, R., et al. 2005, ApJ, 619, 931

Indebetouw, R., Robitaille, T. P., Whitney, B. A., Churchwell, E., Babler, B., Meade, M., Watson, C., \& Wolfire, M. 2007, ApJ, 666,321

Kang, M., Bieging, J. H., Kulesa, C. A., \& Lee, Y. 2009, ApJ, 701,454

Koo, B.-C. 1997, ApJS, 108, 489

Koo, B.-C., \& Moon, D.-S. 1997, ApJ, 475, 194

Koo, B.-C. 1999 , ApJ, 518, 760

Kroupa, P., \& Weidner, C. 2003, ApJ, 598, 1076
Lacy, J. H., et al. 2007, ApJ, 658, L45

Lada, C. J. 1987, Star Forming Regions, 115, 1

Malkov, O. Y. 2007, MNRAS, 382, 1073

Massey, P., \& Hunter, D. A. 1998, ApJ, 493, 180

Massey, P. 2002, ApJS, 141, 81

Mehringer, D. M. 1994, ApJS, 91, 713

Mufson, S. L., \& Liszt, H. S. 1979, ApJ, 232, 451

Ninkov, Z., Bretz, D. R., Easton, R. L., Jr., \& Shure, M. A. 1995, AJ, 110, 2242

Okumura, S.-i., Mori, A., Nishihara, E., Watanabe, E., \&

Yamashita, T. 2000, ApJ, 543, 799

Porras, A., et al. 2007, ApJ, 656, 493

Povich, M. S., et al. 2009, ApJ, 696, 1278

Rana, N. C. 1987, A\&A, 184, 104

Rieke, G. H., et al. 2004, ApJS, 154, 25

Robitaille, T. P., Whitney, B. A., Indebetouw, R., Wood, K., \& Denzmore, P. 2006, ApJS, 167, 256

Robitaille, T. P., Whitney, B. A., Indebetouw, R., \& Wood, K. 2007, ApJS, 169, 328

Robitaille, T. P., et al. 2008, AJ, 136, 2413

Sabbi, E., et al. 2008, AJ, 135, 173

Scalo, J. M. 1986, Fundamentals of Cosmic Physics, 11, 1

Scalo, J. 1998, The Stellar Initial Mass Function (38th

Herstmonceux Conference), 142, 201

Schneps, M. H., Lane, A. P., Downes, D., Moran, J. M., Genzel,

R., \& Reid, M. J. 1981, ApJ, 249, 124

Shepherd, D. S., et al. 2007, ApJ, 669, 464

Simon, J. D., et al. 2007, ApJ, 669, 327

Skrutskie, M. F., et al. 2006, AJ, 131, 1163

Xu, Y., Reid, M. J.,Menten, K. M., Brunthaler, A., Zheng, X. W., \& Moscadelli, L. 2009, ApJ, 693, 413

Whitney, B. A., Indebetouw, R., Bjorkman, J. E., \& Wood, K. 2004, ApJ, 617, 1177

Whitney, B. A., Wood, K., Bjorkman, J. E., \& Cohen, M. 2003a, ApJ, 598, 1079

Whitney, B. A., Wood, K., Bjorkman, J. E., \& Wolff, M. J. 2003b, ApJ, 591, 1049

Whitney, B. A., et al. 2008, AJ, 136, 18

Zapata, L. A., Palau, A., Ho, P. T. P., Schilke, P., Garrod, R. T., Rodríguez, L. F., \& Menten, K. 2008, A\&A, 479, L25 